\title{
Rates of colonization with extended-spectrum $\beta$-lactamase- producing Escherichia coli in Canadian travellers returning from South Asia: a cross-sectional assessment
}

\author{
Gisele Peirano PhD, Daniel B. Gregson MD, Susan Kuhn MD, Otto G. Vanderkooi MD, \\ Diego B. Nobrega DVM, Johann D.D. Pitout MD
}

\section{Abstract}

Background: A previous study in Calgary showed that travel to India was associated with high risk of community-onset infections with extended-spectrum $\beta$-lactamase (ESBL)-producing Escherichia coli. We performed a follow-up study to determine the rate of rectal acquisition of ESBL-producing $E$. coli among travellers to South Asia and to identify the behaviours putting such travellers at high risk for acquiring ESBL-producing E. coli.

Methods: The study was performed at a travel clinic in Calgary. Travellers 18 years or older who were planning to visit South Asia for a period of at least 5 days were included. Three rectal swabs were obtained, and 2 questionaires were administered (before and after travel).

Results: A total of 149 travellers participated between January 2012 and July 2014; of these, 116 (78\%) provided rectal swabs upon return to Calgary and completed both pre- and post-travel questionaires. Of the 109 travellers without colonization with ESBLproducing E. coli upon enrollment, 70 (64\%) acquired ESBL-producing E. coli during travel. Of the 90 participants who visited India, $66(73 \%)$ were positive for ESBL-producing E. coli upon their return to Calgary. Most ESBL-producing E. coli specimens were identified as producing the enzyme CTX-M-15. Behaviours associated with a statistically significant risk of acquiring ESBL-producing E. coli included visiting India (odds ratio [OR] 19.9, 95\% confidence interval [CI] 4.5-88.8), consuming meals with the local population (OR 6.9, 95\% Cl 1.2-39.6), taking any type of antibiotic during travel (OR 4.3, 95\% $\mathrm{Cl} 1.3-14.3$ ) and travelling for any purpose other than business (OR 12.4, 95\% Cl 2.8-55.2).

Interpretation: In this study, travel to India was associated with the highest risk of acquiring ESBL-producing $E$. coli relative to travel to other countries in South Asia. Nonbusiness travel, consuming foods with the local population and the use of antibiotics while travelling were associated with an increased risk of acquiring these antibiotic-resistant organisms while in India. Trial registration: ClinicalTrials. gov, no. NCT01296165.

1 he World Health Organization (WHO) has cited antimicrobial drug resistance as one of the major challenges to global health. ${ }^{1}$ The WHO is especially concerned about the global spread of drug-resistance in Escherichia coli because this species is the most common gram-negative bacterium associated with bloodstream and urinary tract infections in both developed and developing countries. $^{2}$ Before the 2000s, most E. coli strains were susceptible to first-line antibiotics (e.g., cephalosporins and fluoroquinolones), which are often used to treat infections due to E. coli. ${ }^{3}$ However, a 2014 WHO report stated that resistance to the cephalosporins and fluoroquinolones among $E$. coli is widespread, and in many parts of the world, these agents are now ineffective for more than half of patients. ${ }^{1}$ Global resistance to the cephalosporins among E. coli is mainly due to the production of extended-spectrum $\beta$-lactamases (ESBLs), especially the enzyme CTX-M-15. ${ }^{4}$

Since the late 1990s, international travel has grown by about $6 \%$ per year. ${ }^{5}$ According to the International Air Transport Association, ${ }^{6}$ about 1.333 billion passengers were trans-

Competing interests: None declared.

This article has been peer reviewed.

Correspondence to: Johann Pitout, johann.pitout@cls.ab.ca CMAJ Open 2017. DOI:10.9778/cmajo.20170041 
ported over international borders during $2014^{6}$ and about 320 million people visited Asia, northern Africa and the Middle East per year. ${ }^{7}$ Overseas travel as a risk factor for the acquisition of infections due to antibiotic-resistant $E$. coli, including resistance involving ESBLs, is a relatively recent phenomenon. ${ }^{3}$ A previous study from Calgary during the mid-2000s showed that travel to India was associated with high risk of community-onset infection with ESBL-producing $E$. coli among returning travellers. ${ }^{8}$ India is an important overseas destination for Canadians because of migration patterns, and reports have indicated that more than $70 \%$ of E. coli isolated from the Indian community in Canada produce ESBLs. ${ }^{9}$ Therefore, it is conceivable that foreign travel to India potentially plays an important role in the spread of antibiotic resistance, more specifically resistance due to ESBLs, in Canada.

We designed a follow-up study to determine the rate of rectal acquisition of ESBL-producing E. coli among travellers to South Asia (including India) and to identify the potential behaviours putting these travellers at high risk for rectal acquisition of ESBL-producing E. coli.

\section{Methods}

\section{Setting and participants}

The study was performed at the Odyssey Travel and Tropical Medicine Clinic in Calgary, Alberta, which accommodates about 1500-2000 patients per year. This travel clinic provides comprehensive travel medicine services, including preventive vaccinations and prescriptions for antimalarial drugs. Of travellers attending the clinic during the study period, about $20 \%$ were planning travel to visit friends and relatives, about $70 \%$ were tourists, and $10 \%$ were business travellers. The study was performed over a 31-month period (January 2012 to July 2014) with the aim of enrolling 150 participants. The primary outcome measure was the proportion of patients acquiring ESBL, so we did not undertake a formal sample-size calculation. On the basis of previous preliminary data, we estimated that about $40 \%$ of patients (60/150) would acquire ESBL-producing $E$. coli, which would allow us to estimate the risk with a margin of error of 7.8 percentage points. This study was registered with ClinicalTrials. gov, within the Protocol and Results Registration System (registration number NCT01296165).

We did not approach travellers directly to participate in the study. Instead, posters were displayed in the waiting room of the travel clinic asking patients who intended to travel to South Asia to approach a clinic staff member if they were interested in participating in the study. The inclusion criteria were age 18 years or older and planned visit to South Asia (i.e., Afghanistan, Bangladesh, Bhutan, India, Iran, Maldives, Nepal, Pakistan, Sri Lanka) for at least 5 days. We excluded travellers younger than 18 years and those not visiting South Asia. Participants completed a detailed travel itinerary, and we collected a rectal swab specimen before travel. Fourteen couples and a group of 3 who travelled together were given specific instructions on specimen collection and how to avoid cross-contamination.
We asked the participants to return to the travel clinic within 7 days after their return to Canada, at which time they completed a detailed questionnaire about their behaviour and activities during the visit. The completed questionnaires were forwarded to Calgary Laboratory Services and entered into a central Excel database. The following information was requested: main reason or reasons for visiting South Asia, whether the participant was with a tour group, type of area visited (remote, rural, urban or city), participation in camping or swimming (open water or the sea), use of public transport, cost and types of accommodation, consumption of food and beverages, occurrence of diarrhea, consumption of antibiotics and seeking of medical care.

A second rectal swab specimen was obtained within 7 days after each participant's return to Canada, and a third rectal swab specimen was collected 6 months after return. The rectal swabs were immediately submitted to Calgary Laboratory Services and frozen at $-20^{\circ} \mathrm{C}$ until further analysis.

\section{Rectal swab cultures and bacterial isolates}

Rectal swab samples were cultured using chromID-ESBL and chromID-CARBA SMART (Solutions to Manage the Antimicrobial Resistance Threat) selection media (bioMérieux Canada Inc.). Rectal swabs were also inoculated on blood agar plates as a quality control measure, to ensure that stool flora were present within the swab samples. Five colonies per plate were subcultured and identified using matrix-assisted laser desorption ionization-time-of-flight mass spectrometry (Vitek AMS spectrometer, bioMérieux Vitek Systems Inc.).

\section{$\beta$-Lactamase identification and screening for major clones}

We confirmed ESBL production phenotypically using criteria of the Clinical and Laboratory Standards Institute for ESBL screening and disk confirmation tests. ${ }^{10} \mathrm{We}$ determined antimicrobial susceptibility with the VITEK 2 instrument (Vitek AMS; bioMérieux Vitek Systems Inc.). We determined minimum inhibitory concentration for the following drugs: amoxycillin-clavulanic acid, piperacillin-tazobactam, meropenem, ertapenem, amikacin, gentamicin, tobramycin, ciprofloxacin and trimethoprim-sulfamethoxazole. Throughout this study, results were interpreted using the Clinical and Laboratory Standards Institute criteria for broth dilution. ${ }^{10}$

Polymerase chain reaction amplification and sequencing for $b l a_{\mathrm{CTX}-\mathrm{Ms}}, b l a_{\mathrm{OXAs}}, b l a_{\mathrm{TEMs}}, b l a_{\mathrm{SHV}}$ and $b l a_{\mathrm{CMY}}$ genes were carried out on the isolates with a GeneAmp 9700 ThermoCycler instrument (Applied Biosystems) using conditions and primers as previously described. ${ }^{11}$

\section{Statistical analysis}

Participants harbouring ESBL-producing E. coli before departure were excluded from all analyses. For initial selection of risk factors, we used generalized linear mixed-effects models, where the outcome was the presence of ESBLproducing E. coli. Each factor was evaluated individually, and couple-specific random effects were introduced to deal with the hypothetical lack of independence due to isolates 
being obtained from the same couple (or family). We fitted the models to the data via maximum likelihood with 50 quadrature points per scalar using the adaptive GaussHermite quadrature. ${ }^{12}$ Measured predictors (age, duration of travel) were centred at the lowest value. Predictors associated with the estimated logit of harbouring an ESBLproducing $E$. coli at the $15 \%$ significance level were considered for further analyses.

Before performing the multivariable analyses, we tested factors for multicollinearity by looking at nonparametric correlation matrices and the variance inflation factor of all terms. Because of the strong negative correlation between travelling for business purposes and travelling during holiday periods, only the former was retained for further analyses. We evaluated generalized linear mixed-effects models containing different predictors (travel to India, use of antimalarial drugs, travel for nonbusiness purposes, use of public transportation, having meals with the local population and use of any antimicrobials), where the observed effects were initially assumed to be common to all the other terms present in the model. Predictors were dropped one at a time according to their respective $p$ values and confounding of the remaining terms. Factors that were not selected in the initial screening were then added one at a time to verify whether their presence was associated with the outcome, conditional on the presence of the model terms. We tested all possible 2-way interaction terms between the final predictors to evaluate the presence of effect modification. We estimated odds ratios (ORs) with 95\% confidence intervals (CIs) from the final model. For all analyses, there was the implicit assumption that the observed effects were not confounded or modified by variables not considered in the model. Statistical significance was defined as $p<$ 0.05. All analyses were done in $\mathrm{R}$ version 3.4.1.

\section{Ethics approval}

This study was approved by the Conjoint Health Research Ethics Board at the University of Calgary (E-23726).

\section{Results}

Overall, 209 travellers visited the clinic between January 2012 and July 2014 with the intention of travelling to South Asia. Of these, 168 approached the clinic to participate in the study, 149 (71\% of the initial 209) were enrolled, and 116 (78\% of those enrolled) provided rectal swabs upon their return to Canada and completed the pre- and post-travel questionnaires (Figure 1). The remaining 33 travellers who enrolled in the study either cancelled their travel plans $(n=8)$ or did not complete the study requirements (did not provide a second rectal swab on their return to Canada or did not complete the post-travel questionnaire or both) $(n=25)$; these were excluded from further analysis. The characteristics of travellers excluded from analysis are shown in Appendix 1 (available at www.cmajopen.ca/content/5/4/E850/suppl/DC1).

Of the 116 travellers who provided a second rectal swab upon returning to Canada and who completed both pre- and post-travel questionnaires, $7(6 \%)$ had colonization with
ESBL-producing E. coli before leaving Canada (Table 1). Four of these travellers had previously visited India, and 6 of them had colonization with $E$. coli producing CTX-M-15; the remaining isolate was positive for TEM-52. These participants were excluded from the analysis of travel activity associated with acquisition of ESBL-producing Enterobacteriaceae.

The mean age of the other 109 travellers was 34.7 (standard deviation 19.4) years; 71 (65\%) were women, 106 had postsecondary education, 89 had been born in Canada, 3 lived on a rural property or farm, 6 had a history of urinary tract infection within 1 year before travel, 12 had taken antibiotics in the 6 months before travel, 21 had had contact with a health care facility in the 6 months before travel, and 35 had pets. None of these participants had been admitted to hospital within the 6 months before enrollment in the study. The majority of these 109 participants travelled to India ( $n=90[83 \%])$, whereas the remaining travellers visited Nepal $(n=9)$, Pakistan $(n=1)$, Bangladesh $(n=1)$ and Sri Lanka $(n=8)$.

Of the 109 travellers who did not have colonization with ESBL-producing E. coli on enrollment, 70 (64\%) acquired such organisms during travel; only 2 (2\%) acquired AmpCproducing $E$. coli, neither of whom had concurrent colonization with ESBL-producing $E$. coli. None of the travellers had colonization with carbapenem-resistant gram-negative bacteria. Of the 70 participants who acquired ESBL-producing E. coli during travel, 15 (21\%) still had colonization 6 months after their return. At least 1 episode of diarrhea occurred in 68 (62\%) of these 109 travellers; diarrhea was not associated with a statistically increased risk of acquisition of ESBL-producing E. coli (OR 3.12, 95\% CI 0.89-99.01).

Most of the participants who visited India (66/90 [73\%]) were positive for ESBL-producing $E$. coli upon their return to Canada, mainly due to the CTX-M-15 enzyme (58/66 [88\%]). The remaining ESBL types were TEM-26, TEM-52, $\mathrm{SHV}-2$ and SHV-5. A total of 124 isolates were cultured from participants' swab samples. All of these isolates were multidrug-resistant, showing the following levels of nonsusceptibility (i.e., intermediate or complete resistance): $105(85 \%)$ were nonsusceptible to trimethoprimsulfamethoxazole, 80 (65\%) to amoxycillin-clavulanic acid, $37(30 \%)$ to piperacillin-tazobactam, 102 (82\%) to ciprofloxacin, $84(68 \%)$ to tobramycin, $72(58 \%)$ to gentamicin and $7(6 \%)$ to amikacin. No resistance to ertapenem or meropenem was detected. The single traveller to Pakistan and 2 of the 9 who visited Nepal acquired E. coli producing CTX-M15. None of the travellers who visited Sri Lanka acquired ESBL-producing E. coli.

The behaviours that were associated with a statistically significant change in odds of acquiring ESBL-producing $E$. coli are shown in Table 2. Travellers who visited India (OR 19.9, 95\% CI 4.5-88.8), consumed some meals with the local population (OR 6.9, 95\% CI 1.2-39.6), took antibiotics while away (OR 4.3, 95\% CI 1.3-14.3) or travelled for nonbusiness purposes (OR 12.4, 95\% CI 2.8-55.2) were more likely to have colonization with ESBL-producing $E$. coli upon their return to Canada. There was a strong correlation between taking anti- 


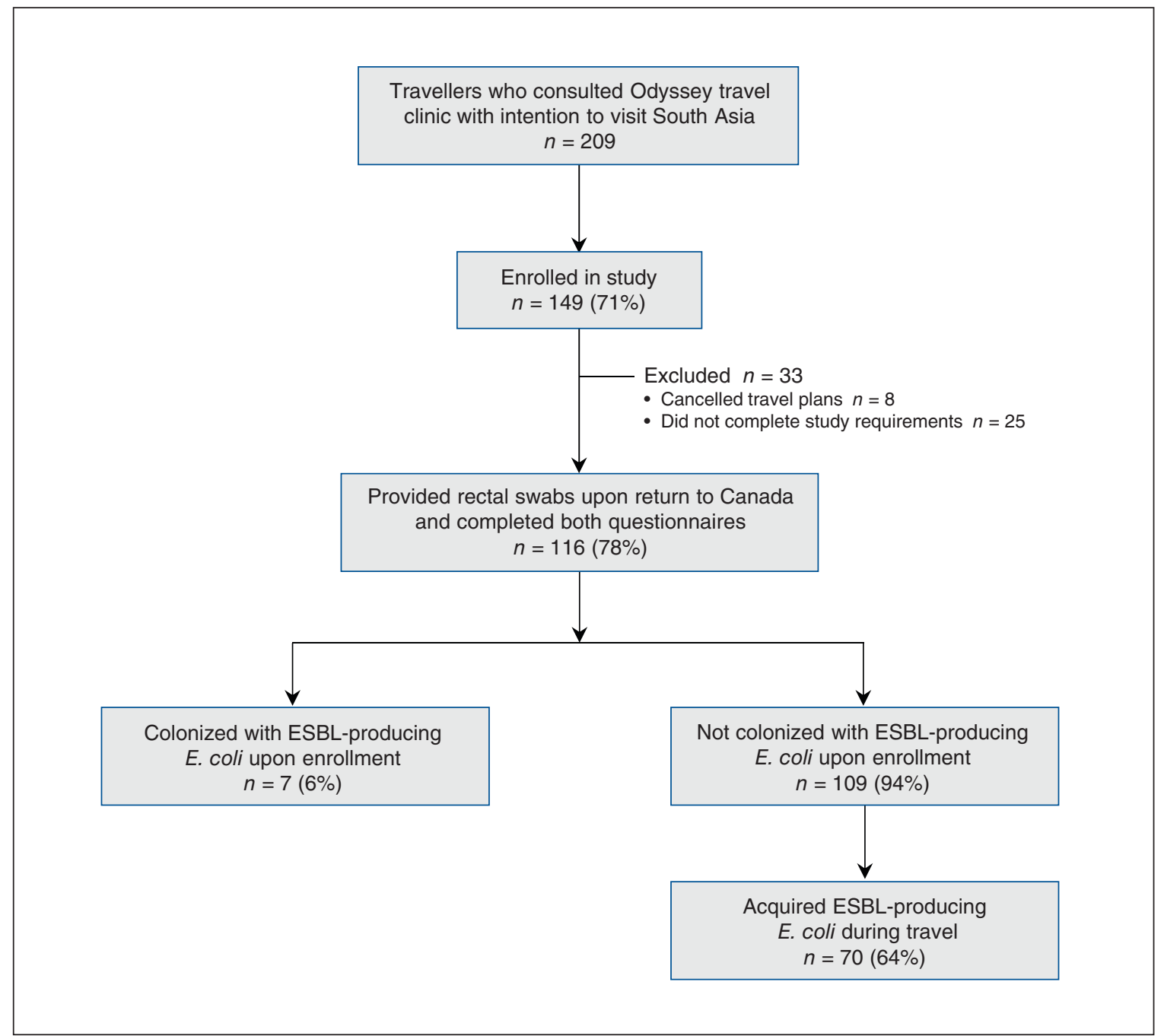

Figure 1: Flow diagram of travellers who participated in the Odyssey travel clinic study. Among those excluded from the analysis, those designated as not completing study requirements did not provide a rectal swab upon their return or did not complete a post-travel questionnaire (or both). In addition to those who acquired ESBL-producing Escherichia coli during travel, 2 travellers acquired AmpC-producing E. coli.

malarial drugs and going to India. Accordingly, we observed a significant association between ESBL colonization and antimalarial drugs in the univariable analyses. Use of public transportation was confounded by other variables that were present in the final model.

\section{Interpretation}

In this study, almost two-thirds of participants acquired multidrug-resistant ESBL-producing E. coli during travel. Of the 90 travellers who visited India, nearly three-quarters were positive for ESBL-producing E. coli upon their return to Calgary, mainly because of CTX-M-15. The behaviours that were associated with statistically significant risk of acquiring ESBL-producing $E$. coli were visiting India specifically, consuming meals with the local population, taking any type of antibiotic during travel and travelling for any purpose other than business. Having diarrhea, eating street food and staying with friends or family members were not significantly associated with ESBL acquisition in our study population.

Visiting South Asia, older age, use of antibiotics during travel (especially the $\beta$-lactams), and gastroenteritis or other gastrointestinal symptoms were previously identified as risk factors for the acquisition of ESBL-producing Enterobacteriaceae. ${ }^{13-15}$ In a study from Finland, traveller's diarrhea (adjusted OR 31.0, 95\% CI 2.7-358.1) and antibiotic therapy for traveller's diarrhea (adjusted OR 3.0, 95\% CI 1.4-6.7) were the most important risk factors for acquiring ESBLproducing E. coli. ${ }^{16}$ The same author group showed that use of loperamide alone to treat mild traveller's diarrhea was not associated with an increased risk of colonization with ESBLproducing $E$. coli. ${ }^{17}$ The largest and most comprehensive travel study investigating multidrug-resistant $E$. coli colonization among returning travellers was performed in the Netherlands. ${ }^{18}$ The authors studied more than 1800 travellers who were negative for ESBL-producing E. coli before travel; of 


\section{Table 1: Demographic, clinical and social characteristics of travellers to South Asia}

\begin{tabular}{|c|c|}
\hline Characteristic & $\begin{array}{c}\text { No. }(\%) \text { of } \\
\text { travellers } \\
(n=116)\end{array}$ \\
\hline Age, yr, mean \pm SD & $36.4 \pm 17.6$ \\
\hline Sex, female & $75(65)$ \\
\hline Education, university & $109(94)$ \\
\hline Canadian-born & $92(79)$ \\
\hline \multicolumn{2}{|l|}{ Premorbid conditions } \\
\hline Diabetes mellitus & $4(3)$ \\
\hline Urinary tract infection ( $<1 \mathrm{yr}$ before travel) & $8(7)$ \\
\hline Other & $5(4)$ \\
\hline \multicolumn{2}{|l|}{ Medications ( 6 mo before travel) } \\
\hline Antibiotics & $13(11)$ \\
\hline Other & $22(19)$ \\
\hline \multicolumn{2}{|c|}{ Contact with health care system (6 mo before travel) } \\
\hline Without hospital admission & $23(20)$ \\
\hline With hospital admission & 0 \\
\hline Residence, rural & $4(3)$ \\
\hline Pets in home & $47(40)$ \\
\hline Travel within previous $6 \mathrm{mo}$ & $27(23)$ \\
\hline Duration of current travel, $\mathrm{d}$, range & 10-38 \\
\hline \multicolumn{2}{|l|}{ ESBL-producing E. coli before travel } \\
\hline Yes & $7(6)$ \\
\hline No & $109(94)$ \\
\hline \multicolumn{2}{|l|}{ Travel destination†‡ } \\
\hline India & $90(83)$ \\
\hline Nepal (Kathmandu, Bhaktapur, Pokhara) & $9(8)$ \\
\hline Pakistan (Lahore) & $1(1)$ \\
\hline Bangladesh (Dhaka) & $1(1)$ \\
\hline Sri Lanka (Colombo, Kandy, Galle) & $8(7)$ \\
\hline \multicolumn{2}{|l|}{ Reason for travel } \\
\hline Tourism & $93(85)$ \\
\hline Visiting friends and relatives & $4(4)$ \\
\hline Business & $12(11)$ \\
\hline With ESBL-producing E. coli after travelł & $70(64)$ \\
\hline To India $(n=90)$ & $66(73)$ \\
\hline To Nepal $(n=9)$ & $2(22)$ \\
\hline To Pakistan $(n=1)$ & $1(100)$ \\
\hline To Bangladesh $(n=1)$ & $1(100)$ \\
\hline To Sri Lanka $(n=8)$ & 0 \\
\hline
\end{tabular}

Note: E. coli $=$ Escherichia coli, $\mathrm{ESBL}=$ extended-spectrum $\beta$-lactamase, $\mathrm{SD}=$ standard deviation.

*Except where indicated otherwise.

†Destination cities in parentheses. For India, destination cities included Agra, Jaipur, Baihar, Amritsar, New Delhi, Mumbai, Pune, Nagpur, Chennai, Kochi, Hyderabad, Kolkata and Panjim.

$\ddagger$ Percentages are based on the 109 participants without ESBL-producing E. coli before travel.
Table 2: Travel activities associated with statistically significant change in odds of acquiring ESBL-producing Escherichia coli on multivariable analysis

\begin{tabular}{|lc|}
\hline Travel activity & $\mathrm{OR}^{*}(95 \% \mathrm{Cl})$ \\
\hline Visited India & $19.9(4.5-88.8)$ \\
\hline Consumed meals with local population & $6.9(1.2-39.6)$ \\
\hline Nonbusiness travel & $12.4(2.8-55.2)$ \\
\hline Use of any antibiotics during travel & $4.3(1.3-14.3)$ \\
\hline $\begin{array}{l}\text { Note: } \mathrm{Cl}=\text { confidence interval, ESBL = extended-spectrum } \beta \text {-lactamase, OR }= \\
\text { odds ratio. } \\
\text { *Based on generalized linear mixed model fit by maximum likelihood; adaptive } \\
\text { Gauss-Hermite quadrature (nAGQ) = 50, Bayesian information criterion = 127.6. }\end{array}$ \\
\hline
\end{tabular}

these, $633(34.3 \%$, CI $32.1 \%-36.5 \%)$ acquired ESBLproducing $E$. coli during international travel. ${ }^{18}$ Of the 181 travellers who visited South Asia, 136 (75.1\%, CI 68.4\%-80.9\%) were positive for ESBL-producing $E$. coli upon their return to the Netherlands. In that study, the strongest predictors for acquisition of ESBL-producing $E$. coli were geographic destination (notably South Asia), antibiotic use during travel, traveller's diarrhea and pre-existing chronic bowel disease. ${ }^{18}$ Eating at street food stalls, consumption of raw vegetables, contact with orphan children, staying in a hostel or guest house, visiting local markets and staying in rural areas were additional strong predictors of colonization with ESBL-producing E. coli. ${ }^{18}$

\section{Limitations}

Our study had some limitations. We included only travellers to South Asia and thus might have missed travellers to other "high-risk" destinations. We made this choice because a previous Calgary study identified visiting India as a risk factor for infection with ESBL-producing E. coli. ${ }^{8}$ Most of the travellers who participated in this study visited India, which might have influenced the high rate of rectal colonization among returning travellers. However, India is an important overseas destination for Canadians because of migration patterns and vacation port of call. The design of the study precluded any guarantee of appropriate randomization. Therefore, there was a risk of selection bias toward travellers to India, and the results of this study might not be applicable to travellers outside South Asia. Our results may have been influenced by volunteer bias, given that those who are more cautious about their health and activities may be more likely to attend a travel clinic and to agree to participate in a study such as this one. One limitation of our analyses was that the OR estimates had low precision. The final model was relatively unstable, which resulted in large standard errors of the estimates and wide confidence intervals for the ORs. Nevertheless, in the logit scale, all standard errors were at least 2-fold lower than the regression estimates.

\section{Conclusion}

In this study, a significant number of Canadian travellers acquired multidrug-resistant ESBL-producing E. coli while visiting India, especially those travelling for reasons other than business, those taking antibiotics while away and those 
who consumed meals with the local population. Therefore, Canadian primary care, emergency and infectious disease physicians should be aware that travellers returning from destinations such as India are likely to have colonization with multidrug-resistant $E$. coli. When such travellers present with bloodstream and urinary tract infections due to gram-negative bacteria, treatment with cephalosporins and fluoroquinolones might not be effective. It is important to counsel patients in the pre-travel setting (e.g., travel clinics).

The implications of our findings for the traveller, the traveller's immediate household and the local community are not well defined at this stage; however, spread of ESBLproducing E. coli (especially those with CTX-M-15) to the local health care and community settings is a distinct possibility. Our results suggest that returning travellers have contributed to the emergence and spread of CTX-M-producing E. coli in Calgary.

\section{References}

1. Antimicrobial resistance: global report on surveillance 2014. Geneva: World Heath Organization; 2014. Available: www.who.int/drugresistance/documents/ surveillancereport/en/ (accessed 2016 Dec. 12).

2. Pitout JD. Extraintestinal pathogenic Escherichia coli: a combination of virulence with antibiotic resistance. Front Microbiol 2012;3:9.

3. van der $\mathrm{Bij} \mathrm{AK}$, Pitout JD. The role of international travel in the worldwide spread of multiresistant Enterobacteriaceae. 7 Antimicrob Chemother 2012;67: 2090-100.

4. Mathers AJ, Peirano G, Pitout JD. The role of epidemic resistance plasmids and international high-risk clones in the spread of multidrug-resistant Enterobacteriaceae. Clin Microbiol Rev 2015;28:565-91.

5. Airline industry overview. Cambridge (MA): Massachusetts Institute of Technology, Global Airline Industry Program. Available: http://web.mit.edu/airlines/ analysis/analysis airline industry.html (accessed 2017 Dec. 6).

6. Annual review 2015. Montréal: International Air Transport Association; 2015. Available: https://www.iata.org/about/Documents/iata-annual-review-2015.pdf (accessed 2016 Dec. 12).

7. UNWTO world tourism barometer. Madrid (Spain): World Tourism Organization. Available: http://mkt.unwto.org/en/barometer (accessed 2016 Dec. 12).

8. Laupland KB, Church DL, Vidakovich J, et al. Community-onset extendedspectrum beta-lactamase (ESBL) producing Escherichia coli: importance of international travel. 7 Infect 2008;57:441-8.

9. Hawser SP, Bouchillon SK, Hoban DJ, et al. Emergence of high levels of extended-spectrum-beta-lactamase-producing gram-negative bacilli in the Asia-Pacific region: data from the Study for Monitoring Antimicrobial Resistance Trends (SMART) program, 2007. Antimicrob Agents Chemother 2009;53: 3280-4.

10. Performance standards for antimicrobial susceptibility testing. CLSI document M100-S20. Wayne (PA): Clinical and Laboratory Standards Institute; 2010.
11. Peirano G, Laupland KB, Gregson DB, et al. Colonization of returning travellers with CTX-M-producing Escherichia coli. 7 Travel Med 2011;18:299-303.

12. Bates D, Mächler M, Bolker B, et al. Fitting linear mixed-effects models using lme4. 7 Stat Softw 2015;67:48.

13. Ostholm-Balkhed A, Tarnberg M, Nilsson M, et al.; Travel Study Group of Southeast Sweden. Travel-associated faecal colonization with ESBL-producing Enterobacteriaceae: incidence and risk factors. 7 Antimicrob Chemother 2013; 68:2144-53.

14. Tängdén T, Cars $\mathrm{O}$, Melhus A, et al. Foreign travel is a major risk factor for colonization with Escherichia coli producing CTX-M-type extended-spectrum beta-lactamases: a prospective study with Swedish volunteers. Antimicrob Agents Chemother 2010;54:3564-8.

15. Weisenberg SA, Mediavilla JR, Chen L, et al. Extended spectrum $\beta$-lactamaseproducing Enterobacteriaceae in international travelers and non-travelers in New York City. PLoS One 2012;7:e45141

16. Kantele A, Laaveri T, Mero S, et al. Antimicrobials increase travelers' risk of colonization by extended-spectrum betalactamase-producing Enterobacteriaceae. Clin Infect Dis 2015;60:837-46.

17. Kantele A, Mero S, Kirveskari J, et al. Increased risk for ESBL-producing bacteria from co-administration of loperamide and antimicrobial drugs for travelers' diarrhea. Emerg Infect Dis 2016;22:117-20.

18. Arcilla MS, van Hattem JM, Haverkate MR, et al. Import and spread of extended-spectrum beta-lactamase-producing Enterobacteriaceae by international travellers (COMBAT study): a prospective, multicentre cohort study. Lancet Infect Dis 2017;17:78-85.

Affiliations: Division of Microbiology (Peirano, Gregson, Pitout), Calgary Laboratory Services; Departments of Pathology and Laboratory Medicine (Peirano, Gregson, Vanderkooi, Pitout), of Medicine (Gregson, Kuhn), of Paediatrics (Kuhn, Vanderkooi) and of Community Health Sciences (Vanderkooi), Cummings School of Medicine, University of Calgary; Department of Production Animal Health (Nobrega), Faculty of Veterinary Medicine, University of Calgary, Calgary, Alta.; Department of Medical Microbiology (Pitout), University of Pretoria, Pretoria, South Africa

Contributors: Daniel Gregson, Susan Kuhn, Otto Vanderkooi and Johann Pitout planned the study. Gisele Peirano, Susan Kuhn, Otto Vanderkooi and Johann Pitout contacted travellers and collected their data. Gisele Peirano performed the laboratory aspects of the study, and Diego Nobrega performed the statistical analysis; Johann Pitout wrote the first draft of the manuscript. All of the authors revised the article for important intellectual content, gave final approval of the version to be published and agreed to be accountable for all aspects of the work.

Funding: This study was funded by Merck \& Co., Inc. Canada (grant number RT630190). The funder had no role in planning the study, collection of data or interpretation of results.

Acknowledgements: The authors are grateful to the staff at the Odyssey Travel and Tropical Medicine Clinic for their assistance with the study.

Supplemental information: For reviewer comments and the original submission of this manuscript, please see www.cmajopen.ca/content/5/4/ E850/suppl/DC1. 